\section{Regression to the Mean Contributes to the Apparent Improvement in Glycemia 3.8 Years After Screening: The ELSA-Brasil Study}

Diabetes Care 2021;44:81-88 | https://doi.org/10.2337/dc20-2030

\section{OBJECTIVE}

Glycemic regression is common in real-world settings, but the contribution of regression to the mean (RTM) has been little investigated. We aimed to estimate glycemic regression before and after adjusting for RTM in a free-living cohort of adults with newly ascertained diabetes and intermediate hyperglycemia (IH).

\section{RESEARCH DESIGN AND METHODS}

The Brazilian Longitudinal Study of Adult Health (ELSA-Brasil) is a cohort study of 15,105 adults screened between 2008 and 2010 with standardized oral glucose tolerance test and $\mathrm{HbA}_{1 \mathrm{c}}$, repeated after $3.84 \pm 0.42$ years. After excluding those receiving medical treatment for diabetes, we calculated partial or complete regression before and after adjusting baseline values for RTM.

\section{RESULTS}

Regarding newly ascertained diabetes, partial or complete regression was seen in 49.4\% (95\% Cl 45.2-53.7); after adjustment for RTM, in 20.2\% (95\% Cl 12.1-28.3). Regarding IH, regression to normal levels was seen in $39.5 \%$ (95\% Cl 37.9-41.3) or in 23.7\% (95\% Cl 22.6-24.3), depending on use of the World Health Organization (WHO) or the American Diabetes Association (ADA) definition, respectively; after adjustment, corresponding frequencies were $26.1 \%$ (95\% Cl 22.4-28.1) and 19.4\% (95\% Cl 18.4-20.5). Adjustment for RTM reduced the number of cases detected at screening: 526 to 94 cases of diabetes, 3,118 to 1,986 cases of WHO-defined IH, and 6,182 to 5,711 cases of ADA-defined IH. Weight loss $\geq 2.6 \%$ was associated with greater regression from diabetes (relative risk 1.52, 95\% $\mathrm{Cl} 1.26-1.84$ ) and $\mathrm{IH}$ (relative risk 1.30, 95\% Cl 1.17-1.45).

\section{CONCLUSIONS}

In this quasi-real-world setting, regression from diabetes at $\sim 4$ years was common, less so for IH. Regression was frequently explained by RTM but, in part, also related to improved weight loss and homeostasis over the follow-up.

Remission from diabetes (1-3) and intermediate hyperglycemia (IH) (4) has been reported in various clinical trials of patients undergoing intensive lifestyle interventions. However, in real-world settings, regression has been little investigated.
Maria Inês Schmidt, ${ }^{1}$ Paula Bracco, ${ }^{1}$

Scheine Canhada, ${ }^{1}$

Joanna M.N. Guimarães, ${ }^{2}$

Sandhi Maria Barreto, ${ }^{3,4}$ Dora Chor, ${ }^{5}$

Rosane Griep, ${ }^{5}$ John S. Yudkin, ${ }^{4}$ and

Bruce B. Duncan ${ }^{1}$
${ }^{1}$ Postgraduate Program in Epidemiology, School of Medicine and Hospital de Clínicas, Universidade Federal do Rio Grande do Sul, Porto Alegre, Rio Grande do Sul, Brazil

${ }^{2}$ National School of Public Health, Fundação Oswaldo Cruz, Rio de Janeiro, Brazil

${ }^{3}$ School of Medicine, Universidade Federal de Minas Gerais, Belo Horizonte, Brazil

${ }^{4}$ Division of Medicine, University College London, London, U.K.

${ }^{5}$ Laboratory of Health and Environment Education, Fundação Oswaldo Cruz, Rio de Janeiro, Brazil

Corresponding author: Maria Inês Schmidt, maria .schmidt@ufrgs.br

Received 15 August 2020 and accepted 10 October 2020

This article contains supplementary material online at $h$ ttps://doi.org/10.2337/figshare.13087271.

(C) 2020 by the American Diabetes Association. Readers may use this article as long as the work is properly cited, the use is educational and not for profit, and the work is not altered. More information is available at https://www.diabetesjournals .org/content/license. 
In one community-based cohort of individuals detected in a trial for screening and early treatment of diabetes, $30 \%$ of the initially detected and confirmed patients presented partial or complete remission from diabetes at 5 years (5). Additionally, in a community-based cohort of individuals with IH (6), remission to normal levels at 1 year was present in $54.0 \%$. Although improvements may be interpreted as related to lifestyle changes, they may be explained, at least partially, by regression to the mean (RTM) (7), owing to the large variability that exists in glucose measures over time (8).

RTM is a statistical phenomenon in which extreme values tend to regress to the mean upon retesting. The Brazilian ELSA-Brasil (Longitudinal Study of Adult Health) study allows evaluation of the frequency of glycemic regression and factors related to it, including RTM. This large contemporary cohort study performed repeat glycemic evaluations by oral glucose tolerance test (OGTT) and $\mathrm{HbA}_{1 \mathrm{c}}$ and advised those who had abnormal values to seek clinical attention and to change lifestyle (9), without performing any additional interventions aimed at changing behavior.

Thus, our objective was to estimate the fraction of ELSA-Brasil participants without known diabetes and meeting diabetes or IH cutoffs at baseline who showed glycemic regression at follow-up, before and after adjustment for RTM, and additionally, to assess the degree to which glycemic regression was associated with improved homeostasis, weight loss, and increased physical activity.

\section{RESEARCH DESIGN AND METHODS}

\section{Study Design and Analytic Sample}

ELSA-Brasil is a prospective occupational cohort study assembled between 2008 and 2010 (visit 1) in six capital cities of different regions of Brazil. We enrolled 15,105 active or retired civil servants, 35 74 years of age, of public universities or research institutions and performed a comprehensive set of questionnaires, clinical measurements, and laboratory tests. We recalled participants between 2012 and 2014 for a new set of interviews and examinations (visit 2), as previously described (10). Ethics committees of each institution approved the research protocols of both visits, and all subjects gave written consent to participate.

\section{Measurements}

Measurements obtained during visits 1 and 2 followed standardized protocols and regular quality control assessments (11). We ascertained age, race/skin color, educational achievement, medication use, and past medical history by standardized questionnaires. Medication use was verified against packages or prescriptions brought to the clinic. Weight, height, and waist circumference were measured with standard techniques, and physical activity was assessed in METminutes/week by the International Physical Activity Questionnaire (IPAC) using the leisure time and transport domains $(10,12)$. BMI was defined as weight $(\mathrm{kg}) /$ height $(\mathrm{m})^{2}$.

We obtained an overnight fasting blood sample by venipuncture soon after arrival at the clinic and followed standardized procedures for a 2-h 75-g OGTT (13). Plasma glucose (PG) was measured using the hexokinase method, insulin by an immunoenzymatic assay (Siemens), and $\mathrm{HbA}_{1 \mathrm{c}}$ by high-pressure liquid chromatography (Bio-Rad), a method certified by the NGSP. Intraclass correlation coefficients expressing between-person variance as a percentage of total variance, obtained analyzing blind replicate pairs of samples drawn at baseline, were 99\% (95\% Cl 0.95-1.0) for glucose and $94 \%(95 \% \mathrm{Cl} 0.86-0.97)$ for $\mathrm{HbA}_{1 \mathrm{c}}$ (14).

We estimated insulin resistance (IR) by HOMA2-IR and $\beta$-cell function by HOMA2- $\beta$ calculations, using the program HOMA Calculator v2.2.3 for values of fasting PG (FPG) within the range of 3$25 \mathrm{mmol} / \mathrm{L}$ and of fasting insulin within the range of 20-400 pmol/L (https://www. dtu.ox.ac.uk/homacalculator/download. php). We calculated the disposition index (DI) as the product of HOMA2- $\beta$ and 1/HOMA2-IR (15).

\section{Ascertainment of Diabetes and IH}

At visit 1, we ascertained diabetes based on laboratory measurements and selfreported information. A participant was considered to have previously diagnosed diabetes when reporting a previous medical diagnosis of diabetes or the use of medication for diabetes in the past 2 weeks. We did not consider as having a previous diagnosis those informing a diagnosis only while pregnant.

Those without a previous diagnosis of diabetes were classified as having diabetes if they reached the thresholds for
FPG ( $\geq 7.0 \mathrm{mmol} / \mathrm{L} ; 126 \mathrm{mg} / \mathrm{dL}), 2 \mathrm{~h}$ PG ( $\geq 11.1 \mathrm{mmol} / \mathrm{L} ; 200 \mathrm{mg} / \mathrm{dL}$ ), or $\mathrm{HbA}_{1 \mathrm{c}}$ ( $\geq 48 \mathrm{mmol} / \mathrm{mol} ; 6.5 \%$ ) $(16,17)$. Among those not meeting diabetes criteria, $\mathrm{IH}$ was defined by both ADA and WHO criteria. For the ADA criteria, we defined impaired glucose tolerance as a 2-h PG $\geq 7.8 \mathrm{mmol} / \mathrm{L}$ ( $\geq 140 \mathrm{mg} / \mathrm{dL}$ ), impaired fasting glucose (IFG) by a FPG $\geq 5.6$ $\mathrm{mmol} / \mathrm{L}$ ( $\geq 100 \mathrm{mg} / \mathrm{dL}$ ), and elevated $\mathrm{HbA}_{1 \mathrm{c}}$ by a value $\geq 39 \mathrm{mmol} / \mathrm{mol}$ ( $\geq 5.7 \%$ ). For the WHO criteria, in addition to impaired glucose tolerance, IFG was defined by a $F P G \geq 6.1 \mathrm{mmol} / \mathrm{L}$ ( $\geq 110 \mathrm{mg} / \mathrm{dL}$ ) and intermediate $\mathrm{HbA}_{1 \mathrm{c}}$ as $\geq 42 \mathrm{mmol} / \mathrm{mol}$ ( $\geq 6.0 \%$ ). At visit 2 we applied the same diagnostic criteria to classify diabetes and $\mathrm{IH}$ and ascertained regression by comparing categories between visits 1 and 2 .

\section{Information Given to Participants} Regarding Abnormal Glucose Values At baseline we provided all laboratory results to participants, alerting that eventual abnormal values in glucose or other tests needed confirmation to establish a clinical diagnosis and that lifestyle changes based on a healthy diet and regular physical activity were important to maintain normal glucose levels and health in general. Being enrolled in a longitudinal study to assess health, participants were aware that new evaluations would take place every 3-4 years.

\section{Definition of Glycemic Regression From Diabetes or IH}

We defined regression from diabetes as being complete (to normal glycemia by all three glycemic tests) or incomplete (to $\mathrm{IH}$, but no longer meeting diabetes criteria by any of the three glycemic tests) (18). We defined regression from IH as normalization of all three glycemic tests.

\section{Analyses}

We calculated incidence of regression from diabetes and $\mathrm{IH}$, and graphically displayed changes in the DI for those who reverted to normal, confirmed $\mathrm{IH}$, or progressed to diabetes.

RTM was initially evaluated graphically by relating baseline with follow-up values using linear regression models. To account for RTM in the evaluation of glycemic changes from baseline to follow-up, we adjusted baseline values with the following formula $(19,20)$ : 


$$
X_{a}=X+(1-r) *(\bar{X}-X)
$$

where:

$X_{a}=$ baseline value adjusted for RTM

$x=$ baseline value

$r=$ correlation between baseline and

follow-up values

$\bar{X}=$ mean baseline value.

This adjustment permitted comparison of glycemic regression before and after these adjustments.

We evaluated the association of baseline characteristics with glycemic regression adjusting through Poisson regression with robust variance for baseline covariates, including age, sex, race/color, educational achievement, family history of diabetes, BMI, waist circumference, physical activity, and glycemic values. The latter were centered at their means. Finally, with similar models, we evaluated the association of larger changes ( $\geq 80$ th percentile) observed during follow-up in weight, waist circumference, physical activity, and the DI with glycemic regression. We fit three progressively adjusted models evaluating the change in exposures, first including age, sex, race/color, and educational achievement (model 1); next, family history of diabetes, baseline BMI, waist circumference, and physical activity (model 2); and finally, baseline fasting and 2-h glucose and $\mathrm{HbA}_{1 \mathrm{c}}$.

We performed all analyses with SAS 9.4 for Windows (SAS Institute, Cary, NC).

\section{RESULTS}

A total of 15,090 participants had complete information to ascertain diabetes at visit $1,13,618$ not reporting a previous diagnosis of diabetes or medication use to treat diabetes during the previous 2 weeks. Among these, 950 met diabetes criteria, 7,165 met IH criteria by at least one laboratory test, and 5,503 had normoglycemic values. After additional exclusions, including those with a history of bariatric surgery or use of diabetes medication between visits, our analytic sample was composed of 526 subjects with newly diagnosed diabetes, 6,182 of $\mathrm{IH}$ and 4,937 of normoglycemia, thus totaling 11,645 participants (Supplementary Fig. 1).

Among these 11,645 participants free of known diabetes at baseline and not receiving medical treatment, there were slightly fewer men (44.8\%) than women (55.2\%), most (66.9\%) were between 45 and 64 years old, White $(53.6 \%)$, with a university degree (55.5\%), overweight or obesity $(59.7 \%)$, and without a family history of diabetes (64.3\%). Among the 526 participants with newly ascertained diabetes at baseline, there were slightly more men (54.9\%) than women (45.1\%), participants being most frequently aged 45-64 years ( $72.4 \%)$, White (42.1\%), with less than a university degree (58.1\%), and with overweight or obesity (80.2\%). Similar characteristics were seen for the 6,182 subjects with $\mathrm{IH}$ at baseline, although the proportions of men and women were closer, and subjects tended to be younger and with less obesity. From crude and initial descriptive information, it is apparent that the cohort gained weight, increased physical activity, and worsened glycemic values between baseline and follow-up. Of note as well, mean FPG at baseline was at the level of the ADA cutoff to define IH (Supplementary Table 1).

As seen in Table 1, after a mean of 3.84 \pm 0.42 years of follow-up, among those ascertained as having diabetes at baseline, we found substantial glycemic regression (to IH or to normal levels) when considering the results of each test separately: $41.3 \%$ (95\% Cl 34.6-48.0), 41.5\% (95\% Cl 35.7-47.3), and $70.4 \%(95 \% \mathrm{Cl}$ 63.3-77.4) for FPG, 2-h PG, and $\mathrm{HbA}_{1 \mathrm{c}}$, respectively. Regression to normal levels was considerably less frequent, $2.9 \%$, $7.9 \%$, and $24.1 \%$, respectively. We also examined results of multiple tests at a single occasion to identify those less likely to regress from diabetes upon retesting. On the basis of having two positive tests, only $10.0-17.5 \%$ regressed, complete regression to normoglycemia being virtually absent. With three positive tests, only $11.6 \%$ regressed, none to normal levels.

As also seen in Table 1, regression from $\mathrm{IH}$ varied according to the criteria used to define it. When we used the WHO criteria, regression to normal levels was seen in $44 \%, 43.8 \%$, and $80 \%$ for FPG, $2-h$ $\mathrm{PG}$, and $\mathrm{HbA}_{1 \mathrm{c}}$, respectively, and when we defined by the ADA criteria, in $27.0 \%$, $43.8 \%$, and $61.3 \%$, respectively. Multiple testing also reduced the likelihood of regression, although less effectively than when used for detecting diabetes.

Scatter plots of baseline and follow-up glycemic values for the three tests (Fig. 1) illustrate far-from-perfect correlations ( $r=0.62$ for FPG and 2-h PG, and $r=$ 0.46 for $\left.\mathrm{HbA}_{1 \mathrm{c}}\right)$ and downward inclinations ( $\beta=0.84,0.75$ and 0.54 for FPG, 2-h $\mathrm{PG}$, and $\mathrm{HbA}_{1 \mathrm{c}}$, respectively) of the estimated regression line from the diagonal, indicative of RTM.

To evaluate the importance of RTM in overall regression, we estimated the frequency of glycemic regression after accounting for RTM. To do so, we adjusted baseline glycemic values according to the equation described in Research Design and Methods, here defining diabetes and $\mathrm{IH}$ when detected by at least one of the three tests. Figure 2 shows these results, on the left depicting categories based on the observed glycemic values at baseline and on the right, categories based on those adjusted to account for RTM.

Among the 526 participants (top circles) meeting diabetes cutoffs at baseline, 260 (49.4\%; 95\% Cl 45.2-53.7) regressed: 234 (44.5\%; 95\% Cl 40.0-38.1) to IH and 26 (4.9\%; 95\% Cl 3.1-6.8) to normoglycemia. After adjustment for RTM, the frequency of diabetes at baseline decreased substantially, with only 94 of 526 of the initially defined participants (17.9\%) now meeting diabetes cutoffs. Glycemic regression was still present, albeit of lower proportions: 19 (20.2\%; 95\% Cl 12.1-28.3) regressed, 17 (18.1\%; $95 \% \mathrm{Cl} 10.3-25.9)$ to $\mathrm{IH}$ and 2 (2.0\%; 95\% Cl 0-5.0) to normal.

Among the 3,118 subjects (middle circles) meeting WHO IH cutoffs at baseline, 1,234 (39.5\%; 95\% Cl 37.9-41.3) regressed, reaching normal values, and 404 progressed to diabetes $(13.0 \% ; 95 \% \mathrm{Cl}$ 11.8-14.1). After adjustment for RTM, 1,986 of 3,118 of the initially defined participants $(63.7 \%)$ now met IH cutoffs, with 519 (26.1\%; 95\% Cl 22.4-28.1) regressing to normal and 492 progressing to diabetes (24.3\%; 95\% Cl 22.4-26.2).

Among the 6,182 subjects (bottom circles) meeting the ADA criteria for $\mathrm{IH}$, 1,464 (23.7\%; 95\% Cl 22.6-24.3) regressed, reaching normal values, and 499 progressed to diabetes $(8.1 \% ; 95 \% \mathrm{Cl}$ 7.4-8.7). After adjustments, 5,711 of 6,182 of the initially defined participants (92.4\%) now met IH cutoffs, with 1,110 (19.4\%; 95\% Cl 18.4-20.5) regressing to normal and 649 progressing to diabetes (11.4\%; 95\% Cl 10.5-12.2).

Thus, after accounting for RTM, the fraction of those regressing decreased 
Table 1-Glycemic regression at 3.8 years of follow-up of those meeting cutoffs for diabetes or IH at baseline

\begin{tabular}{|c|c|c|c|c|c|c|c|}
\hline \multirow[b]{2}{*}{ Diabetes at baseline } & \multirow{2}{*}{$\frac{\text { All }(N=526)}{N}$} & \multicolumn{2}{|c|}{ Any regression } & \multicolumn{2}{|c|}{$\begin{array}{c}\text { Regression } \\
\text { to IH }\end{array}$} & \multicolumn{2}{|c|}{ Regression to normal } \\
\hline & & $N$ & $\%$ & $N$ & $\%$ & $N$ & $\%$ \\
\hline \multicolumn{8}{|l|}{$\begin{array}{l}\text { Multiple tests on the same occasion: FPG, 2-h PG, and/or } \\
\mathrm{HbA}_{1 \mathrm{c}} \\
\text { Abnormal by one of the three tests }\end{array}$} \\
\hline $\mathrm{FPG} \geq 7.0 \mathrm{mmol} / \mathrm{L}$ & 206 & 85 & 41.3 & 79 & 38.4 & 6 & 2.9 \\
\hline 2-h PG $\geq 11.1 \mathrm{mmol} / \mathrm{L}$ & 277 & 115 & 41.5 & 93 & 33.6 & 22 & 7.9 \\
\hline $\mathrm{HbA}_{1 \mathrm{c}} \geq 48 \mathrm{mmol} / \mathrm{mol}$ & 162 & 114 & 70.4 & 75 & 46.3 & 39 & 24.1 \\
\hline \multicolumn{8}{|l|}{ Abnormal by two tests } \\
\hline FPG $\geq 7.0$ and $2-h$ PG $\geq 11.1 \mathrm{mmol} / \mathrm{L}$ & 97 & 17 & 17.5 & 16 & 16.5 & 1 & 1 \\
\hline FPG $\geq 7.0$ and $\mathrm{HbA}_{1 \mathrm{c}} \geq 48 \mathrm{mmol} / \mathrm{mol}$ & 50 & 7 & 14 & 7 & 14 & 0 & 0 \\
\hline 2-h PG $\geq 11.1 \mathrm{mmol} / \mathrm{L}$ and $\mathrm{HbA}_{1 \mathrm{c}} \geq 48 \mathrm{mmol} / \mathrm{mol}$ & 50 & 5 & 10 & 5 & 10 & 0 & 0 \\
\hline \multirow[t]{2}{*}{ Abnormal by all three tests } & 43 & 5 & 11.6 & 5 & 11.6 & 0 & 0 \\
\hline & All $(N=3,118)$ & & & & & \multicolumn{2}{|c|}{ Regression to normal } \\
\hline IH at baseline defined by the WHO criteria & $N$ & & & & & $N$ & $\%$ \\
\hline \multicolumn{8}{|l|}{$\begin{array}{l}\text { Multiple tests on the same occasion: FPG, 2-h PG, and/or } \\
\qquad \mathrm{HbA}_{1 c} \\
\text { Abnormal by one of the three tests }\end{array}$} \\
\hline FPG $\geq 6.1 \mathrm{mmol} / \mathrm{L}$ & 1,078 & & & & & 474 & 44 \\
\hline 2-h PG $\geq 7.8 \mathrm{mmol} / \mathrm{L}$ & 2,031 & & & & & 889 & 43.8 \\
\hline $\mathrm{HbA}_{1 \mathrm{c}} \geq 42 \mathrm{mmol} / \mathrm{mol}$ & 603 & & & & & 485 & 80.4 \\
\hline \multicolumn{8}{|l|}{ Abnormal by two tests } \\
\hline $\mathrm{FPG} \geq 6.1$ and $2-\mathrm{h} P G \geq 7.8 \mathrm{mmol} / \mathrm{L}$ & 457 & & & & & 85 & 18.6 \\
\hline FPG $\geq 6.1$ and $\mathrm{HbA}_{1 \mathrm{c}} \geq 42 \mathrm{mmol} / \mathrm{mol}$ & 127 & & & & & 46 & 36.2 \\
\hline 2-h PG $\geq 7.8 \mathrm{mmol} / \mathrm{L}$ and $\mathrm{HbA}_{1 \mathrm{c}} \geq 42 \mathrm{mmol} / \mathrm{mol}$ & 124 & & & & & 38 & 30.7 \\
\hline \multirow[t]{2}{*}{ Abnormal by all three tests } & 66 & & & & & 7 & 10.6 \\
\hline & All $(N=6,182)$ & & & & & \multicolumn{2}{|c|}{ Regression to normal } \\
\hline IH at baseline defined by the ADA criteria & $N$ & & & & & $N$ & $\%$ \\
\hline
\end{tabular}

Multiple tests on the same occasion: FPG, 2-h PG, and/or $\mathrm{HbA}_{1 \mathrm{c}}$

Abnormal by one of the three tests

FPG $\geq 5.5 \mathrm{mmol} / \mathrm{L}$

4,811

2,031

1,678

$\mathrm{HbA}_{1 \mathrm{c}} \geq 38.8 \mathrm{mmol} / \mathrm{mol}$

1,344

939

338

336

$\begin{array}{cc}1,298 & 27 \\ 889 & 43.8 \\ 1,028 & 61.3 \\ & \\ 154 & 11.5 \\ 142 & 15.1 \\ 72 & 21.3 \\ 19 & 5.7\end{array}$

Data are based on FPG, 2-h PG, and $\mathrm{HbA}_{1 \mathrm{c}}$ in ELSA-Brasil, 2008-2020.

substantially (59.1\%; from $49.4 \%$ to 20.2\%) for participants initially labeled as having diabetes; moderately (34.0\%) for those labeled as having $\mathrm{IH}$ by the WHO cutoffs, and less so (17.9\%) for those labeled as having IH by the ADA cutoffs.

We then investigated whether changes in glucose homeostasis accompanied glycemic regression during the followup. From a mean DI at baseline of 47.4 (Supplementary Table 1), for those ascertained as having diabetes at baseline (left panels, Supplementary Fig. 2) who regressed to normal, the median $\mathrm{DI}$ increased by $11.3(P<0.001)$, with improvements in both $\beta$-cell function and insulin sensitivity. For those regressing to
$\mathrm{IH}$, the median DI increased less (6.8\%; $P<0.001$ ), with more modest improvement in insulin sensitivity and a minor worsening in $\beta$-cell function. For those with diabetes confirmed at reevaluation, DI decreased $(-5.3 ; P<0.001)$, reflecting worsened $\beta$-cell function and stable insulin sensitivity (Supplementary Fig. 2).

Similarly, among those classified as having $\mathrm{IH}$ at baseline (Supplementary Fig. 2 , right panels), from a mean baseline DI of 65.4 , for those who regressed to normoglycemia, the median DI increased slightly $(4.3 ; P<0.001)$, with both improved insulin sensitivity and $\beta$-cell function. For those who confirmed IH, DI decreased somewhat $(-3.0 ; P<0.001)$, and for those who progressed to diabetes, the median DI decreased $(-13.0 ; P<$ 0.001 ), with $\beta$-cell function and insulin sensitivity both worsened.

We also evaluated whether changes in lifestyle between visits could explain part of the regression from diabetes and $\mathrm{IH}$ among those not reporting bariatric surgery and antidiabetic medication at follow-up. We ran three separate sets of progressive models, each one evaluating three changes that occurred between visits - weight loss, reduced waist circumference, and increased physical activity. As seen in Table 2, in the final adjusted model, the likelihood of regression from diabetes, compared with those 

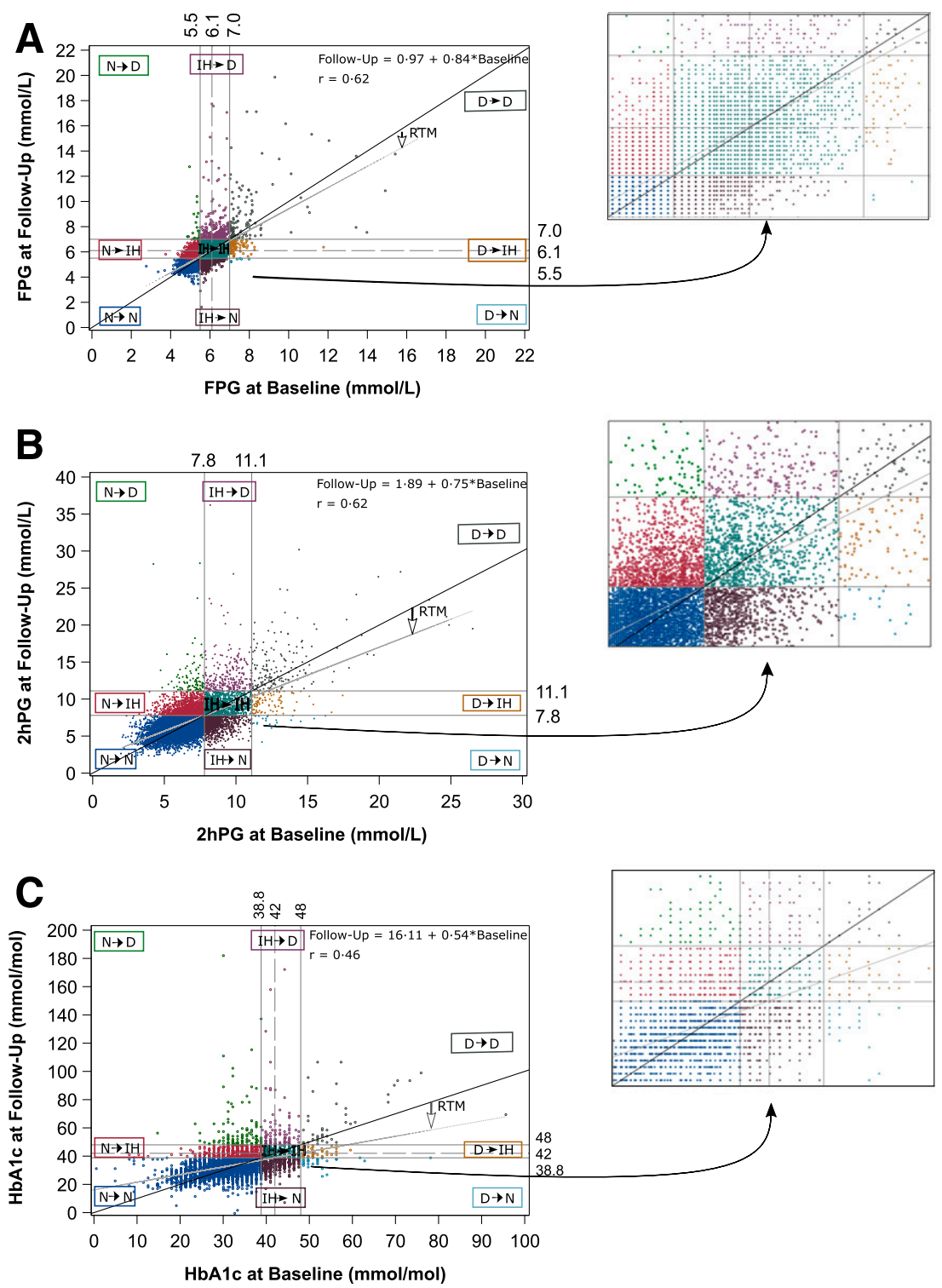

Figure 1-Scatter plots of baseline and follow-up glycemic values and estimated parameters of linear regression for FPG $(A), 2-\mathrm{h} \mathrm{PG}(B)$, and glycated hemoglobin $\left(\mathrm{HbA}_{1 \mathrm{c}}\right)(C)$. The dark line is the $45^{\circ}$ diagonal; the fine line is the predicted fit. Additional graphs show details of central portions. $D$, diabetes; $\mathrm{N}$, normal.

with lesser or unchanged values, was greater for those with a larger $(\geq 80$ percentile) change between visits: a $\geq 2.6 \%$ loss of initial weight (relative risk [RR] 1.52; 95\% Cl 1.26-1.84), a $\geq 1.4 \mathrm{~cm}$ decrease in waist circumference (RR 1.33; 95\% Cl 1.07-1.67); and a $\geq 552$ MET-min/ week increase in physical activity (RR 1.29; $95 \% \mathrm{Cl} 1.08-1.55)$.

The likelihood of regression from $\mathrm{IH}$ was greater in the final model only for those with a larger weight loss (RR 1.30; 95\% $\mathrm{Cl} 1.17-1.45)$ and a larger reduction in waist circumference (RR $1.22 ; 95 \% \mathrm{Cl}$ 1.08-1.38).

In similar models, a larger improvement in the $\mathrm{DI}$ ( $\geq 12.5$; $\geq 80$ percentile) was associated with regression from diabetes (RR 1.91; 95\% Cl 1.63-2.23) and IH (RR 2.84; 95\% Cl 2.60-3.09).

\section{CONCLUSIONS}

Within the context of a cohort study of adults tested with an OGTT and an $\mathrm{HbA}_{1 \mathrm{c}}$, at 3.8 years of follow-up, $49.4 \%$ of those ascertained as having diabetes had regressed (15.4\% to normal and 34\% to $\mathrm{IH})$. After adjustment for RTM, regression decreased considerably, with only $20.2 \%$ regressing, $5 \%$ to normal and $15 \%$ to $\mathrm{IH}$. Among those ascertained as having $\mathrm{IH}$, regression was also frequent when defined by WHO criteria, $39.5 \%$ and $26.1 \%$ after adjustment for RTM; but less so when defined by the ADA criteria, $23.7 \%$, and $19.4 \%$ after adjustment for RTM. The use of more than one test helped identify those more likely to regress. To our knowledge, this is the first evaluation of RTM among individuals with new-onset diabetes and $\mathrm{IH}$ detected in a large and contemporary observational study and receiving no structured intervention.

The regression from diabetes we found after adjusting for RTM $(20 \%)$ is comparable to that of those enrolled in a trial of community-based screening (Anglo-Danish-Dutch Study of Intensive Treatment In People with Screen Detected Diabetes in Primary Care [ADDITION]Cambridge study), in which $30 \%$ of participants initially detected were below diagnostic cutoffs $\left(\mathrm{HbA}_{1 \mathrm{c}}<6.5 \%\right.$; $<43$ $\mathrm{mol} / \mathrm{mol}$ off antidiabetic medication) at the 5-year follow-up (5). Their slightly greater frequency of glycemic regression may be due to the more intensive intervention offered in the treatment arm of the trial. On the other hand, both our and the ADDITION results show a greater frequency of regression than that seen in an integrated health care delivery system, which found diabetes remission at 7 years under usual care in only $4.6 \%$ of individuals, probably in part due to less focus on identifying these patients (21).

The regression from IH we found before adjustment for RTM (27.0-80.4\%, depending on the criteria used for FPG, 2-h PG, or $\mathrm{HbA}_{1 \mathrm{c}}$ ) (Table 1 ) is comparable to that found for $\mathrm{IH}$ in the CRONICAS (Center of Excellence in Chronic Diseases) cohort study (31.4-66.9\% depending on the diagnostic criteria used for FPG and $\mathrm{HbA}_{1 \mathrm{c}}$ ) at 2.2 years. Although they estimated RTM to be small, the contribution of RTM to regression to normal was not evaluated (22). Our results are also comparable to those found in the ADDITION-Leicester study, which followed up at 1-year individuals with IH (defined by a single OGTT with WHO cutoffs) in a community screening program, finding that $54.0 \%$ had regressed to normal levels.

Of note, the adjustment for RTM we adopted here resulted in a marked reduction $(59.1 \%)$ in the fraction of diabetes cases regressing, a moderate one (34.0\%) when considering WHO-defined $\mathrm{IH}$, and a discrete one (17.9\%) for ADAdefined IH. That the effect of RTM is more pronounced among subjects with diabetes and WHO-defined $\mathrm{IH}$ is consonant 
Diabetes

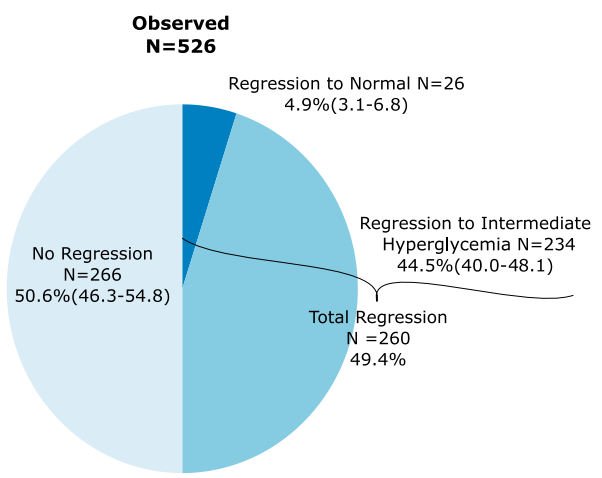
After accounting for $\mathrm{RTM}$
$\mathrm{N}=94$

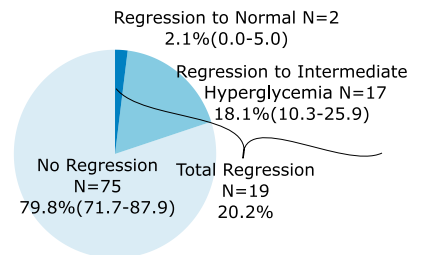

Intermediate Hyperglycemia (WHO)

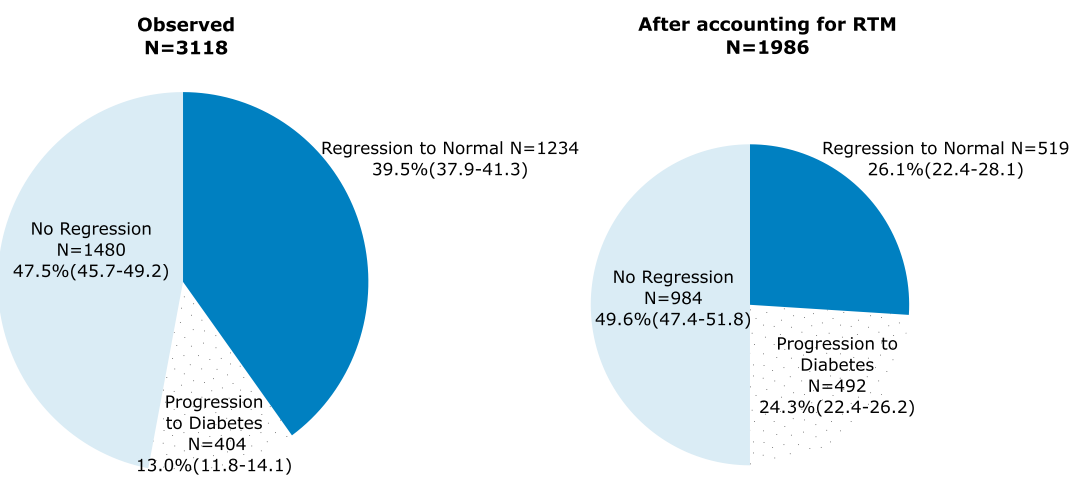

Intermediate Hyperglycemia (ADA)

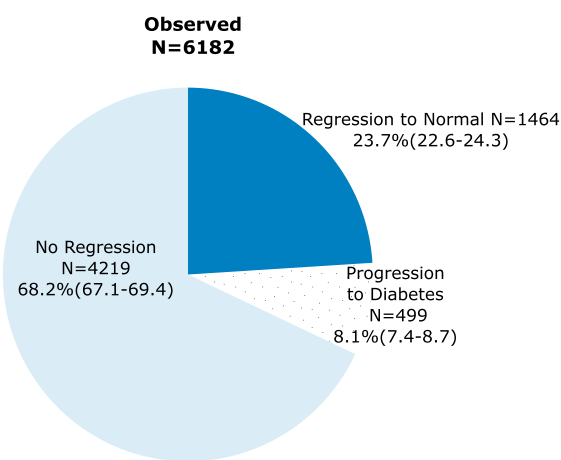

After accounting for RTM $\mathbf{N}=\mathbf{5 7 1 1}$

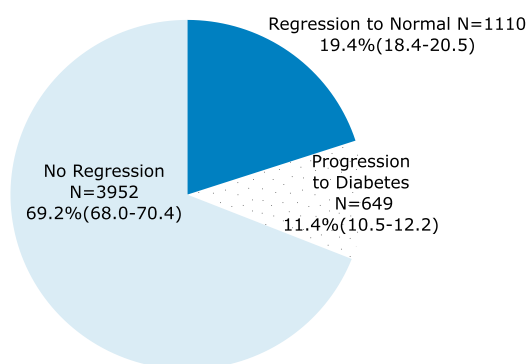

Figure 2-Proportions of participants with diabetes and $\mathrm{IH}$ at baseline and their glycemic regression at follow-up, before and after adjustments for RTM. Top, diabetes at baseline. Bottom, $\mathrm{IH}$ at baseline. The size of the circles illustrates the relative reduction in baseline cases after accounting for RTM.

with the understanding that those with more extreme initial values are more likely to have larger RTM upon retesting. Of note also, adjustment for RTM and the use of multiple tests in the same occasion, although reducing the number of individuals likely to regress from diabetes or $\mathrm{IH}$, also reduces substantially the number of subjects ascertained at screening as having diabetes or IH. Important implications of these findings merit discussion.
First, the substantial proportion of subjects meeting diabetes cutoffs at baseline regressing upon retesting reiterates the need to confirm a positive test before labeling diabetes, as recommended in current clinical guidelines. Although clinicians in busy day-to-day practice may skip confirmation, our findings illustrate that lack of confirmation will result in a large proportion of individuals receiving pharmacological treatment who do not really have diabetes.
That $34.6 \%$ of those reporting diabetes in a recent National Health and Nutrition Examination Survey (NHANES) survey presented $\mathrm{HbA}_{1 \mathrm{c}}$ values of $\leq 6.5 \%$ suggests that some degree of overdiagnosis may be present (23). Additionally, it is possible that the fraction of success achieved in clinical trials aiming for diabetes remission will be somewhat exaggerated if diabetes-level glycemia off medication is not confirmed before randomization. In settings where a confirmatory test on a subsequent occasion is not viable, our findings support the use of multiple tests for the diagnosis of diabetes, an option now included in the ADA recommendations $(24,25)$.

Second, our findings further document that the prevalence and incidence of diabetes and $\mathrm{IH}$, when based on a single test, overestimate true rates. In fact, taking the short-term between-day variability of FPG into account in NHANES led to an estimated $24 \%$ lower prevalence of diabetes (8). Yet major groups, such as the Non-Communicable Disease Risk Factor Collaboration (NCD-RisC) (26), the Global Burden of Disease Study (27), and the International Diabetes Federation Atlas (28), which characterize prevalence of diabetes worldwide, do not make adjustments for this problem or discuss its relevance. Using multiple tests on the same occasion (Table 1) may be useful to avoid errors in both epidemiologic studies and clinical trials.

Third, our findings among those with IH showing glycemic regression related to RTM or to small changes in lifestyle, as discussed later, highlight the importance of confirming $\mathrm{IH}$ before prescribing a structured diabetes prevention program or a medication to prevent diabetes.

Of note also, our finding of glycemic regression not explained by medical treatments after adjustment for RTM in a quasi-real-world scenario is also novel and merits discussion. The glycemic regression we found after taking RTM into account could be interpreted as due to changes in lifestyle during follow-up. Associations of glycemic regression with $>5 \%$ weight loss and an improved DI support this interpretation and give further evidence that simple, real-world interventions have significant metabolic benefits for diabetes prevention (29). Whether increased insulin sensitivity and/or improved $\beta$-cell function underlie these changes deserves further 
Table 2-Poisson regression with robust variance estimates of the associations between changes from baseline and regression from diabetes and IH

\begin{tabular}{|c|c|c|c|c|c|}
\hline \multicolumn{2}{|c|}{ Model 1} & \multicolumn{2}{|c|}{ Model 2} & \multicolumn{2}{|c|}{ Model 3} \\
\hline RR & $95 \% \mathrm{Cl}$ & $\mathrm{RR}$ & $95 \% \mathrm{Cl}$ & RR & $95 \% \mathrm{Cl}$ \\
\hline
\end{tabular}

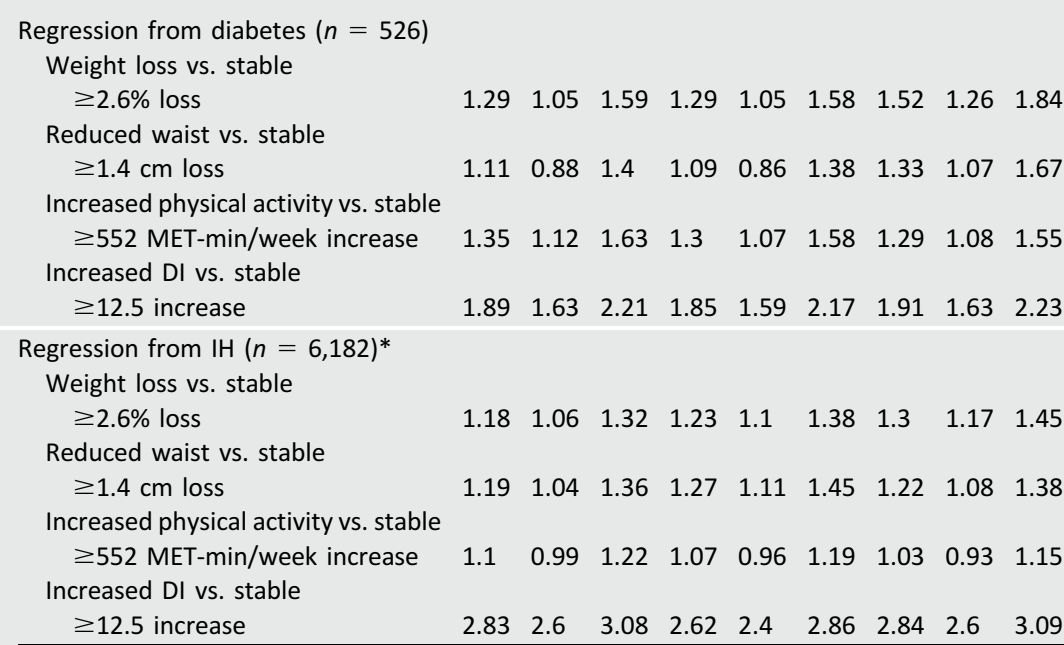

Data are shown after 3.8 years of follow-up among those not reporting bariatric surgery or initiating medication for diabetes in ELSA-Brasil, 2008-2014. Model 1, weight loss, reduced waist, increased physical activity, or DI plus age, sex, race/color, educational achievement, and family history of diabetes. Model 2 , model 1 plus baseline BMI, waist circumference, and physical activity. Model 3, model 2 plus centered baseline FPG and 2-h PG, and $\mathrm{HbA}_{1 \mathrm{c}} .{ }^{*} \mathrm{IH}$ based on the ADA criteria.

evaluation, particularly regarding IFG, which is less likely to improve with standard lifestyle changes (30).

A final note regarding the definition used here for glycemic regression: For diabetes, we considered partial or complete regression as a decrease below standard diagnostic cutoffs, FPG $(<7.0$ $\mathrm{mmol} / \mathrm{L} ; 126 \mathrm{mg} / \mathrm{dL})$, 2-h PG $(<11.1$ $\mathrm{mmol} / \mathrm{L} ; 200 \mathrm{mg} / \mathrm{dL})$, or $\mathrm{HbA}_{1 \mathrm{c}}(<48$ $\mathrm{mmol} / \mathrm{mol} ; 6.5 \%)$. For complete regression from diabetes, we required a 2-h $\mathrm{PG}<7.8 \mathrm{mmol} / \mathrm{L}(140 \mathrm{mg} / \mathrm{dL})$, an FPG $<5.6 \mathrm{mmol} / \mathrm{L}(100 \mathrm{mg} / \mathrm{dL})$, or an $\mathrm{HbA}_{1 \mathrm{c}}$ $<39 \mathrm{mmol} / \mathrm{mol}(5.7 \%)$, unless analyses referred specifically to changes based on the WHO criteria, for which cutoffs for FPG and $\mathrm{HbA}_{1 \mathrm{c}}$ are higher. There is no consensus yet on what should be considered "normal values" for each test. Our use of the most stringent ADA definition underestimates glycemic regression when based on the WHO (16) or National Institute for Health and Care Excellence (NICE) cutoffs (31).

Limitations to our findings need to be considered. First, because we excluded approximately one-third of those ascertained as having diabetes at baseline due to subsequent medical treatment, frequencies of regression observed are probably overestimates. However, even if none of those treated would have regressed, the frequency of regression would remain large.

Second, when generalizing our results, population mean glycemic values must be considered. Given the high mean glycemia in our sample, RTM would be greater in other populations in which $\mathrm{IH}$ and diabetes represent a smaller fraction of the whole.

Third, because our study was observational, with greater or lesser lifestyle change occurring without investigator input, it does not permit ascribing changes in glycemia directly to specific changes in behavior/lifestyle. However, that we also observed regression from hypertension related to the modest weight losses during the same time frame (32) further supports the contention that unstructured and low-intensity real-world lifestyle interventions can exert clinically relevant change. In the absence of a control group and considering the small weight changes observed, there remains the possibility of our findings being explained by residual confounding and or incomplete adjustment for RTM.

Strengths of our findings also deserve attention. ELSA-Brasil is a large contemporary cohort, with standardized measurements, low attrition, and glycemia assessed with all three standard diagnostic tests. Our analyses provided adjustments for RTM in the evaluation of glycemic regression from diabetes and $\mathrm{IH}$, which to our knowledge have rarely been conducted within the context of observational studies in the field of diabetes.

In conclusion, after an average of 3.8 years, in those without medical treatment, glycemic regression had occurred in many of those meeting diabetes and IH cutoffs at baseline. RTM accounted for an important part of this regression. Some of the remaining regression probably resulted from modest weight loss achieved during follow-up.

Funding. This study was funded by the Brazilian Ministry of Health (Science and Technology Department) and the Brazilian Ministry of Science, Technology and Innovation (FINEP and CNPq). Grant numbers: 01060010.00 and 01.10.0643. 03 (RS), 01060212.00 and 01.10.0742-00 (BA), 01060300.00 and 01.12 .0284 .00 (ES), 0106 0278.00 and 0110074600 (MG), 01060115.00 and $01.10 .0773-00$ (SP), and 01060071.00 and 01.11.0093.01 (RJ). This study was financed in part by the Coordenação de Aperfeiçoamento de Pessoal de Nível Superior-Brasil (CAPES) and the Fundação de Amparo a Pesquisa do Rio Grande do Sul (IATS/FAPERGS).

Duality of Interest. No potential conflicts of interest relevant to this article were reported. Author Contributions. M.I.S. drafted the report. M.I.S., P.B., J.S.Y., and B.B.D. designed the analytic plan. M.I.S., S.M.B., D.C., R.G., and B.B.D. are principal investigators of ELSA-Brasil. P.B. and S.C. conducted the data analysis. M.I.S., P.B., S.C., J.M.N.G., S.M.B., D.C., R.G., J.S.Y., and B.B.D. interpreted the data and made meaningful revisions to the manuscript. M.I.S. is the guarantor of this work and, as such, had full access to all the data in the study and takes responsibility for the integrity of the data and the accuracy of the data analysis.

Prior Presentation. Initial results were presented in part as a poster at the 2019 American Heart Association EPI|Lifestyle Scientific Sessions, Houston, Texas, 5-8 March, 2019.

\section{References}

1. Gregg EW, Chen H, Wagenknecht LE, et al.; Look AHEAD Research Group. Association of an intensive lifestyle intervention with remission of type 2 diabetes. JAMA 2012;308:2489-2496

2. Esposito K, Maiorino Ml, Petrizzo M, Bellastella $G$, Giugliano D. The effects of a Mediterranean diet on the need for diabetes drugs and remission of newly diagnosed type 2 diabetes: follow-up of a randomized trial. Diabetes Care 2014;37:18241830

3. Lean ME, Leslie WS, Barnes AC, et al. Primary care-led weight management for remission of type 2 diabetes (DiRECT): an open-label, clusterrandomised trial. Lancet 2018;391:541-551 4. Perreault L, Pan Q, Mather KJ, Watson KE, Hamman RF, Kahn SE; Diabetes Prevention Program Research Group. Effect of regression from prediabetes to normal glucose regulation on 
long-term reduction in diabetes risk: results from the Diabetes Prevention Program Outcomes Study. Lancet 2012;379:2243-2251

5. Dambha-Miller H, Day AJ, Strelitz J, Irving G, Griffin SJ. Behaviour change, weight loss and remission of Type 2 diabetes: a communitybased prospective cohort study. Diabet Med 2020;37:681-688

6. Bodicoat DH, Khunti K, Srinivasan BT, et al. Incident Type 2 diabetes and the effect of early regression to normoglycaemia in a population with impaired glucose regulation. Diabet Med 2017;34:396-404

7. Thomas DM, Clark N, Turner D, et al. Best (but oft-forgotten) practices: identifying and accounting for regression to the mean in nutrition and obesity research. Am J Clin Nutr 2020;111:256265

8. Selvin E, Crainiceanu CM, Brancati FL, Coresh J. Short-term variability in measures of glycemia and implications for the classification of diabetes. Arch Intern Med 2007;167:15451551

9. Schmidt MI, Bracco PA, Yudkin JS, et al. Intermediate hyperglycaemia to predict progression to type 2 diabetes (ELSA-Brasil): an occupational cohort study in Brazil. Lancet Diabetes Endocrinol 2019;7:267-277

10. Schmidt MI, Duncan BB, Mill JG, et al. Cohort profile: Longitudinal Study of Adult Health (ELSABrasil). Int J Epidemiol 2015;44:68-75

11. Schmidt MI, Griep RH, Passos VM, et al. Strategies and development of quality assurance and control in the ELSA-Brasil. Rev Saude Publica 2013;47(Suppl. 2):105-112 [in Portuguese] 12. Aquino EML, Barreto SM, Bensenor IM, et al. Brazilian Longitudinal Study of Adult Health (ELSA-Brasil): objectives and design. Am J Epidemiol 2012;175:315-324

13. World Health Organization. Definition, Diagnosis and Classification of Diabetes Mellitus and Its Complications: report of a WHO consultation. Geneva, Switzerland, World Health Organization, 1999. Accessed 9 April 2014.
Available from https://whqlibdoc.who.int/hq/ 1999/who_ncd_ncs_99.2.pdf

14. Ladwig R, Vigo A, Fedeli LMG, et al. Variability in baseline laboratory measurements of the Brazilian Longitudinal Study of Adult Health (ELSA-Brasil). Braz J Med Biol Res 2016; 49:e5381

15. Retnakaran R, Shen S, Hanley AJ, Vuksan V, Hamilton JK, Zinman B. Hyperbolic relationship between insulin secretion and sensitivity on oral glucose tolerance test. Obesity (Silver Spring) 2008;16:1901-1907

16. World Health Organization. Definition and Diagnosis of Diabetes Mellitus and Intermediate Hyperglycaemia: Report of a WHO/IDF Consulation. Geneva, Switzerland, World Health Organization, 2006. Accessed 10 March 2014. Available from https://www.who.int/diabetes/publications/ diagnosis_diabetes2006/en/

17. American Diabetes Association. Standards of medical care in diabetes-2014. Diabetes Care 2014;37(Suppl. 1):S14-S80

18. Buse JB, Caprio S, Cefalu WT, et al. How do we define cure of diabetes? Diabetes Care 2009; 32:2133-2135

19. Rocconi LM, Ethington CA. Assessing longitudinal change: adjustment for regression to the mean effects. Res High Educ 2009;50:368-376 20. Shepard DS, Finison LJ. Blood pressure reductions: correcting for regression to the mean. Prev Med 1983;12:304-317

21. Karter AJ, Nundy S, Parker MM, Moffet $\mathrm{HH}$ Huang ES. Incidence of remission in adults with type 2 diabetes: the diabetes \& aging study. Diabetes Care 2014;37:3188-3195

22. Lazo-Porras M, Bernabe-Ortiz A, Ruiz-Alejos $A$, et al. Regression from prediabetes to normal glucose levels is more frequent than progression towards diabetes: the CRONICAS Cohort Study Diabetes Res Clin Pract 2020;163:107829

23. CDC.National Diabetes Statistics Report 2020. Estimates of Diabetes and Its Burden in the United States. Atlanta, GA, US Department of Health and Human Services, 2020, pp. 32
24. American Diabetes Association. 2. Classification and diagnosis of diabetes: Standards of Medical Care in Diabetes-2019. Diabetes Care 2019;42(Suppl. 1):S13-S28

25. Selvin E, Wang D, Matsushita K, Grams ME, Coresh J. Prognostic implications of single-sample confirmatory testing for undiagnosed diabetes: a prospective cohort study. Ann Intern Med 2018;169:156-164

26. NCD Risk Factor Collaboration (NCD-RisC) Worldwide trends in diabetes since 1980: a pooled analysis of 751 population-based studies with 4.4 million participants. Lancet 2016 387:1513-1530

27. GBD 2017 Risk Factor Collaborators. Global, regional, and national comparative risk assessment of 84 behavioural, environmental and occupational, and metabolic risks or clusters of risks for 195 countries and territories, 1990-2017: a systematic analysis for the Global Burden of Disease Study 2017. Lancet 2018;392:1923-1994 28. International Diabetes Federation. IDF Diabetes Atlas, 9th edition, 2019. Accessed 2 August 2020. Available from https://www.diabetesatlas .org/en/

29. Galaviz KI, Weber MB, Straus A, Haw JS, Narayan KMV, Ali MK. Global diabetes prevention interventions: a systematic review and network meta-analysis of the real-world impact on incidence, weight, and glucose. Diabetes Care 2018;41:1526-1534

30. Campbell MD, Sathish T, Zimmet PZ, et al. Benefit of lifestyle-based T2DM prevention is influenced by prediabetes phenotype. Nat Rev Endocrinol 2020;16:395-400

31. NICE. Overview. Type 2 diabetes: prevention in people at high risk: guidance [Internet]. NICE. Accessed 3 August 2020. Available from https:// www.nice.org.uk/guidance/ph38

32. Guimarães JMN, Griep RH, Fonseca MJM, et al. Four-year adiposity change and remission of hypertension: an observational evaluation from the Longitudinal Study of Adult Health (ELSABrasil). J Hum Hypertens 2020;34:68-75 